\title{
DIRICHLET PROBLEM IN LIPSCHITZ DOMAINS WITH BMO DATA
}

\author{
EUGENE B. FABES AND UMBERTO NERI
}

\begin{abstract}
In any bounded starlike Lipschitz domain, the harmonic functions whose boundary values are in BMO (the class of functions with bounded mean oscillation) of the boundary are shown to be characterized by the property that their Littlewood-Paley measures are Carleson measures. This result extends the analogous characterization found by the authors when the domain in question is a half-space.
\end{abstract}

1. Introduction and statement of the result. The purpose of this paper is to characterize, by means of certain Carleson measures, all harmonic functions in a Lipschitz domain which have boundary values with bounded mean oscillation. This work extends the 2-dimensional result in [6], obtained with the aid of conformal mapping, and utilizes several results from Dahlberg's papers ([1]-[4]). We acknowledge with pleasure the helpful conversations with Professor B. E. J. Dahlberg on this subject matter. Corresponding results for half-spaces were the precursors of this paper (see [5] and [6]).

Let us fix our notation and definitions. $D$ denotes a bounded domain in $\mathbf{R}^{n+1}$ whose boundary $\partial D$ is a Lipschitz manifold of dimension $n \geqslant 2$. For a technical reason explained below, we shall assume that $D$ is star-shaped with respect to some point $P_{0} \in D$. Throughout the paper, $P$ will denote points inside $D$ while $Q$ denotes points on $\partial D$. The letter $C$ will denote various positive constants depending only on the Lipschitz character and on the dimension of $\partial D$.

Along $\partial D$ we have an inward-pointing vector field of nontangential unit vectors $\nu=\nu(Q)$ in $\mathbf{R}^{n+1}$. For each $Q_{0} \in \partial D$ and $\delta>0$, the intersection of $\partial D$ with the circular cylinder having for axis the line spanned by $\nu\left(Q_{0}\right)$ and radius $\delta$ is called a surface ball with center $Q_{0}$ and radius $\delta$. It is denoted by $B_{\delta}=B_{\delta}\left(Q_{0}\right)$, or simply by $B$. The portion of the cylinder given by

$$
T(B)=\left\{Q+r \nu\left(Q_{0}\right): Q \in B_{\delta}\left(Q_{0}\right), 0 \leqslant r \leqslant 2 \delta\right\}
$$

will be called a (Carleson) tube over $B$. A positive measure $\mu$ is a Carleson measure in $D$ if there exists a constant $A>0$ such that, for all balls $B=B_{\delta}\left(Q_{0}\right)$

$$
\mu[T(B)] \leqslant A \sigma(B)
$$

where $\sigma$ denotes the (Lebesgue) surface measure on $\partial D$. If the tube $T(B)$ is

Received by the editors January 30, 1979.

AMS (MOS) subject classifications (1970). Primary 31B25, $35 \mathrm{~J} 05$.

(c) 1980 American Mathematical Society 0002-9939/80/0000-0009/\$02.75 
contained in a coordinate neighborhood of $D$ with coordinates $(x, t) \in \mathbf{R}^{n} \times$ $\mathbf{R}$, we have the representations:

$$
\begin{aligned}
Q_{0} & =\left(x_{0}, \varphi\left(x_{0}\right)\right) \in \partial D, \\
B & =B_{\delta}\left(Q_{0}\right)=\left\{(x, \varphi(x)):\left|x-x_{0}\right| \leqslant \delta\right\}, \\
T(B) & =\left\{(x, t):\left|x-x_{0}\right| \leqslant \delta \text { and } 0 \leqslant t-\varphi(x) \leqslant 2 \delta\right\}
\end{aligned}
$$

for some real valued function $\varphi$ uniformly Lipschitz continuous on $\mathbf{R}^{n}$.

For any $f \in L^{2}(\partial D)$ and any surface ball $B$ as above, we denote by

$$
f_{B}=\sigma(B)^{-1} \int_{B} f d \sigma
$$

the integral average of $f$ over $B$. If the quasi-norm

$$
\|f\|_{*}=\sup _{B}\left\{\sigma(B)^{-1} \int_{B}\left|f-f_{B}\right|^{2} d \sigma\right\}^{1 / 2}
$$

is finite, we say that $f$ has bounded mean oscillation on $\partial D$ (with respect to surface measure) and we write $f \in \operatorname{BMO}(\partial D)$, or $\operatorname{BMO}(\partial D, d \sigma)$ if necessary.

For each $P \in D$, we let $\omega_{P}$ denote the harmonic measure evaluated at $P$. It is well known that each $\omega_{P}$ is a probability measure on $\partial D$ and is absolutely continuous with respect to $d \sigma$. Hence,

$$
d \omega_{P}(Q)=K(P, Q) d \sigma(Q)
$$

and each nonnegative density $K(P, \cdot)$ has integral one on $\partial D$. More recently, Dahlberg has proved in [1] that each $K(P, \cdot)$ is square integrable on $\partial D$ and that, for all surface balls $B$, it satisfies the condition

$$
\left\{\sigma(B)^{-1} \int_{B} K^{2}(P, \cdot) d \sigma\right\}^{1 / 2} \leqslant B_{2} \sigma(B)^{-1} \int_{B} K(P, \cdot) d \sigma
$$

for some constant $B_{2}=B_{2}(P)>0$ depending only on the point $P$ and the Lipschitz character of the boundary. It is clear from this that each $f \in$ $L^{2}(\partial D)$, in particular $f \in \operatorname{BMO}(\partial D)$, has harmonic extension

$$
[\mathscr{P}(f)](P)=\int_{\partial D} f d \omega_{P}=\int_{\partial D} f(Q) K(P, Q) d \sigma(Q)
$$

for all $P \in D$, called the Poisson integral of $f$ for the domain $D$. This harmonic function in $D$ has almost everywhere $(d \sigma)$ nontangential limits equal to $f$ on $\partial D[\mathbf{1}]$.

With each harmonic function $u$ in $D$ we associate the (Littlewood-Paley) measure $\mu$ given by

$$
d \mu(P)=|\nabla u(P)|^{2} \rho(P) d P
$$

where $\rho(P)$ is the distance from $P$ to $\partial D$ and $d P$ is the $(n+1)$-dimensional Lebesgue measure.

Definition. The linear space of all harmonic functions $u$ in $D$ such that, for every surface ball $B$,

$$
\|u\|_{* *}=\sup _{B}[u]_{B}<\infty
$$


where

$$
[u]_{B}^{2}=\sigma(B)^{-1} \mu[T(B)]=\sigma(B)^{-1} \int_{T(B)} d \mu(P)
$$

is denoted by $\mathrm{HMO}(D)$ (see [5] and [6]).

In other words, $\mathrm{HMO}(D)$ is the class of harmonic functions whose Littlewood-Paley measures are also Carleson measures in $D$. Our main result is as follows.

TheOREM. A function $u$ is in $\operatorname{HMO}(D)$ if and only if $u=\mathscr{P}(f)$ for some $f \in \operatorname{BMO}(D)$. Moreover, $\|f\|_{*} \approx\|u\|_{* *}$.

The proof of this result depends on the lemma below. In the proof of the lemma, we use an estimate from [7] which requires that $D$ be star-shaped. This geometric restriction appears nowhere else in our paper.

From now on, $B=B_{\delta}$ is a surface ball of radius $\delta, B^{*}$ is its concentric double, and $Z$ denotes the center point of the tube $T(B)$.

LEMMA. There exists a constant $C>0$ independent of $\delta$ such that, for all $f \in \mathrm{BMO}(\partial D)$,

$$
v(Z) \equiv \int_{\partial D \backslash B^{*}}\left|f-f_{B^{*}}\right| K(Z, Q) d \sigma(Q)<C\|f\|_{*} .
$$

Proof. Letting $\omega=\omega_{P_{0}}$, the harmonic measures of $D$ evaluated at the star-center $P_{0}$, we set

$$
f_{\omega_{0}}=\omega\left(B^{*}\right)^{-1} \int_{B^{*}} f d \omega
$$

Likewise, we set

$$
f_{\omega_{j}}=\omega\left(B_{j}\right)^{-1} \int_{B_{j}} f d \omega
$$

where the $B_{j}$ are surface balls of radius $2^{j} \delta$ concentric with $B$, and we let $S_{j}=B_{j} \backslash B_{j-1}$, with $B_{1}=B^{*}$ and integers $j \geqslant 2$. We note that

$$
v(Z) \leqslant\left|f_{B^{*}}-f_{\omega_{0}}\right|+\int_{\partial D \backslash B^{*}}\left|f-f_{\omega_{0}}\right| K(Z, Q) d \sigma(Q) .
$$

By Schwarz's inequality and condition $\left(B_{2}\right)$, we see that

$$
\begin{aligned}
\left|f_{\omega_{0}}-f_{B^{*}}\right| \leqslant & \omega\left(B^{*}\right)^{-1} \int_{B^{*}}\left|f-f_{B^{*}}\right| K\left(P_{0}, Q\right) d \sigma(Q) \\
\leqslant & \frac{\sigma\left(B^{*}\right)}{\omega\left(B^{*}\right)}\left\{\sigma\left(B^{*}\right)^{-1} \int_{B^{*}} K^{2}\left(P_{0}, Q\right) d \sigma\right\}^{1 / 2} \\
& \times\left\{\sigma\left(B^{*}\right)^{-1} \int_{B^{*}}\left|f-f_{B^{*}}\right|^{2} d \sigma\right\}^{1 / 2} \\
\leqslant & \frac{B_{2}}{\omega\left(B^{*}\right)}\left\{\int_{B^{*}} K\left(P_{0}, Q\right) d \sigma\right\}\|f\|_{*}=B_{2}\|f\|_{*}
\end{aligned}
$$

where $B_{2}$ depends only on $P_{0}$. 
The integral over $\partial D \backslash B^{*}=\cup S_{j}$ is dominated by

$$
\sum_{j} \int_{S_{j}}\left|f-f_{\omega_{j}}\right| K(Z, Q) d \sigma+\sum_{j}\left|f_{\omega_{j}}-f_{\omega_{0}}\right| \int_{S_{j}} K(Z, Q) d \sigma=\mathrm{I}+\mathrm{II} \text {. }
$$

Multiplying and dividing by $K\left(P_{0}, Q\right)$ and using the estimate [7, Lemma 4]

$$
\underset{Q \in S_{j}}{\text { ess } \sup _{j}}\left[\frac{K(Z, Q)}{K\left(P_{0}, Q\right)}\right] \leqslant c_{j} \omega\left(B_{j}\right)^{-1}
$$

where $c_{j}=O\left(2^{-\lambda_{j}}\right)$ is independent of $\delta($ and $\lambda>0)$, we obtain

$$
\mathrm{I} \leqslant \sum_{j} c_{j}\left\{\omega\left(B_{j}\right)^{-1} \int_{B_{j}}\left|f-f_{\omega_{j}}\right| d \omega\right\} .
$$

Likewise, each term in II is dominated by

$$
j c_{j}\left[\sup _{k}\left|f_{\omega_{k}}-f_{\omega_{k-1}}\right|\right] \omega\left(B_{j}\right)^{-1} \omega\left(S_{j}\right)
$$

with

$$
\begin{aligned}
\left|f_{\omega_{k}}-f_{\omega_{k-1}}\right| & =\omega\left(B_{k-1}\right)^{-1}\left|\int_{B_{k}-1}\left(f-f_{\omega_{k}}\right) d \omega\right| \\
& \leqslant \frac{\omega\left(B_{k}\right)}{\omega\left(B_{k-1}\right)}\left\{\omega\left(B_{k}\right)^{-1} \int_{B_{k}}\left|f-f_{\omega_{k}}\right|_{d \omega}\right\} .
\end{aligned}
$$

It is well known [7, p. 311] that $\omega\left(2 B_{k-1}\right) \leqslant c \omega\left(B_{k-1}\right)$ with $c$ independent of $k$ and $\delta$. In addition, as noted in [8] for more general densities, the supremum of the harmonic BMO means

$$
\omega(B)^{-1} \int_{B}\left|f-f_{\omega(B)}\right| d \omega
$$

is equivalent to the supremum of the corresponding means relative to $d \sigma$ and hence to the $\operatorname{BMO}(\partial D)$ means previously defined. Consequently, we obtain the bounds

$$
I \leqslant c_{0}\|f\|_{*} \text { and }\left|f_{\omega_{k}}-f_{\omega_{k-1}}\right| \leqslant c_{1}\|f\|_{*}
$$

with $c_{0}$ and $c_{1}$ independent of $\delta$ and $k$. Hence,

$$
\mathrm{II} \leqslant c_{1}\|f\|_{*}\left(\sum_{j} j c_{j}\right)
$$

and estimate (6) follows.

2. Proof of the theorem. We begin with the necessity part of the statement. The condition $u \in \operatorname{HMO}(D)$ implies the finiteness of the integral $\int_{D}|\nabla u(P)|^{2} \rho(P) d P$. By a result of Dahlberg [3, p. 4], we have the estimate

$$
\int_{\partial D}\left\{N\left[u-u\left(P_{0}\right)\right](Q)\right\}^{2} d \sigma \leqslant C_{0} \int_{D}|\nabla u(P)|^{2} \rho(P) d P
$$

for any fixed $P_{0} \in D$, where $N[\cdot]$ denotes the nontangential maximal opera- 
tor. It follows then that $u$ has nontangential limits $f(Q)$ for a.e. $Q \in \partial D$ (see [1] and also [7]). Moreover, $f \in L^{2}(\partial D)$ and (see [2]) $u=\mathscr{P}(f)$.

On the other hand, with $D$ replaced by a tube $T(B)$ and with $P_{0}=Z$, the center of $T(B)$, a dilation shows that the constant $C_{0}$ in (7) is independent of the point $Z$. Hence, we have

$$
\begin{aligned}
\int_{B}|f(Q)-u(Z)|^{2} d \sigma(Q) & \leqslant \int_{\partial T(B)}|u-u(Z)|^{2} d \sigma \\
& \leqslant C \int_{T(B)}|\nabla u(P)|^{2} \operatorname{dist}(P, \partial T(B)) d P \\
& \leqslant C \int_{T(B)}|\nabla u(P)|^{2} \rho(P) d P .
\end{aligned}
$$

Therefore, dividing by $\sigma(B)$, we see that $f \in \operatorname{BMO}(\partial D)$ with

$$
\|f\|_{*} \leqslant C\|u\|_{* *} \text {. }
$$

Passing to the sufficiency, we choose any $f \in \operatorname{BMO}(\partial D)$ and we shall examine its Poisson integral. Fixing any surface ball $B$ with radius $\delta$ and subtracting from $f$ its mean value over the concentric double $B^{*}$, we form the usual splitting:

$$
\begin{aligned}
f-f_{B^{*}} & =\left[f-f_{B^{*}}\right] \chi_{B^{*}}+\left[f-f_{B^{*}}\right]\left(1-\chi_{B^{*}}\right) \equiv f_{1}+f_{2}, \\
u & =\mathscr{P}\left(f-f_{B^{*}}\right)=\mathscr{P}\left(f_{1}\right)+\mathscr{P}\left(f_{2}\right) \equiv u_{1}+u_{2} .
\end{aligned}
$$

Then, by (4), (5), and [3, p. 4], we see that

$$
\begin{aligned}
\sigma(B)\left[u_{1}\right]_{B}^{2} & \leqslant \int_{D}\left|\nabla u_{1}(P)\right|^{2} \rho(P) d P \\
& \leqslant C^{2} \int_{\partial D}\left|f_{1}\right|^{2} d \sigma=C^{2} \int_{B^{*}}\left|f-f_{B^{*}}\right|^{2} d \sigma
\end{aligned}
$$

and hence

$$
\left[u_{1}\right]_{B} \leqslant C\|f\|_{*}
$$

Considering separately the positive and negative parts of $f_{2}$ and applying Harnacks's inequality to each of the corresponding positive harmonic functions we obtain the estimate

$$
\begin{aligned}
\left|\nabla u_{2}(P)\right| & \leqslant C \rho(P)^{-1} \int_{\partial D}\left|f_{2}(Q)\right| K(P, Q) d \sigma(Q) \\
& \equiv C \rho(P)^{-1} v_{2}(P) .
\end{aligned}
$$

Without loss of generality, we may assume that $B$ is centered at the origin and that $T(B)$ has local coordinates $(x, t)$, with $t>\varphi(x)$, and center $Z=$ $(0, \delta)$. Since, by estimate $(6), v_{2}(Z) \equiv\left[\mathscr{P}\left(\left|f_{2}\right|\right)\right](Z) \leqslant C\|f\|_{*}$ while $G(5 Z, Z)$ $>c \delta^{1-n}$, the Comparison Theorem for positive harmonic functions [4, Lemma 3] implies the bound

$$
v_{2}(P) \leqslant C\|f\|_{*} \delta^{n-1} G(5 Z, P)
$$


for all $P \in T(B)$, with some new $C>0$. Moreover, by virtue of the same Comparison Theorem, on all of $T(B)$ we can dominate $G(5 Z, P)$ by $G_{T\left(5 B^{*}\right)}(5 Z, P)$, the Green's function for the tube $T\left(5 B^{*}\right)$ with pole at the point $5 Z$. Therefore, combining these estimates with (9), we conclude that

$$
\left|\nabla u_{2}(P)\right| \leqslant C\|f\|_{*} \delta^{n-1} \rho(P)^{-1} G_{T\left(5 B^{*}\right)}(5 Z, P)
$$

for all $P \in T(B)$.

In view of (10), the quantity $\left[u_{2}\right]_{B}^{2}$ is dominated by

$$
\|f\|_{*}^{2} \delta^{-n} \int_{|x|<\delta} \int_{\varphi(x)}^{\varphi(x)+2 \delta}[t-\varphi(x)]^{-1}\left\{\delta^{n-1} G_{T\left(5 B^{*}\right)}(5 Z ;(x, t))\right\}^{2} d t d x .
$$

If we scale our variables $x \in \mathbf{R}^{n}$ and $t \in \mathbf{R}$ by letting $(x, t)=\delta(\tilde{x}, \tilde{t})$ and we set $\varphi_{\delta}(\tilde{x})=\delta^{-1} \varphi(\delta x)$, then the previous expression becomes

$$
\|f\|_{*}^{2} \int_{|\tilde{x}|<1} \int_{\varphi_{\delta}(\tilde{x})}^{\varphi_{\delta}(\tilde{x})+2}\left[\tilde{t}-\varphi_{\delta}(\tilde{x})\right]^{-1}\left\{\delta^{n-1} G_{T\left(5 B^{*}\right)}(5 Z ;(\delta \tilde{x}, \delta \tilde{t}))\right\}^{2} d \tilde{t} d \tilde{x}
$$

This scaling dilates $T\left(5 B^{*}\right)$ to the new tube

$$
\tilde{T}=\left\{(\tilde{x}, \tilde{t}):|\tilde{x}| \leqslant 5,0 \leqslant \tilde{t}-\varphi_{\delta}(\tilde{x}) \leqslant 10\right\} .
$$

Moreover, by uniqueness of the Green's function, $\tilde{T}$ has Green's function $\tilde{G}$ satisfying

$$
\tilde{G}((0,5) ;(\tilde{x}, \tilde{t}))=\delta^{n-1} G_{T\left(5 B^{*}\right)}((0,5 \delta) ;(\delta \tilde{x}, \delta \tilde{t}))
$$

for all $(\tilde{x}, \tilde{t}) \in \tilde{T}$. Hence, substituting this formula in the previous integral, we obtain the estimate

$$
\left[u_{2}\right]_{B}^{2} \leqslant C^{2}\|f\|_{*}^{2} \int_{|\tilde{x}|<1} \int_{\varphi_{\delta}(\tilde{x})}^{\varphi_{\delta}(\tilde{x})+2} \frac{\{\tilde{G}((0,5) ;(\tilde{x}, \tilde{t}))\}^{2}}{\tilde{t}-\varphi_{\delta}(\tilde{x})} d \tilde{t} d \tilde{x}
$$

Now let $\tilde{Z}=(0,5)$, let $\tilde{\omega}$ denote the harmonic measure on $\tilde{T}$ evaluated at $\tilde{Z}$, and let $\tilde{B}$ denote a surface ball on $\partial \tilde{T}$ with center $\left(\tilde{x}, \varphi_{\delta}(\tilde{x})\right)$ and radius $\left[\tilde{t}-\varphi_{\delta}(\tilde{x})\right]$. Applying Lemma 1 of [1] we obtain

$$
\begin{aligned}
\tilde{G}(\tilde{Z} ;(\tilde{x}, \tilde{t})) & \leqslant C\left[\tilde{t}-\varphi_{\delta}(\tilde{x})\right]^{1-n} \tilde{\omega}(\tilde{B}) \\
& \leqslant C\left[\tilde{t}-\varphi_{\delta}(\tilde{x})\right] \tilde{\boldsymbol{\sigma}}(\tilde{B})^{-1} \int_{\tilde{B}} \tilde{K}(\tilde{Z}, \cdot) d \tilde{\sigma}
\end{aligned}
$$

where $\tilde{K}$ is the Poisson kernel for the tube $\tilde{T}$. Finally,

$$
\tilde{G}(\tilde{Z} ;(\tilde{x}, \tilde{t})) \leqslant C\left[\tilde{t}-\varphi_{\delta}(\tilde{x})\right] \Re[\tilde{K}(\tilde{Z}, \cdot)]\left(\tilde{x}, \varphi_{\delta}(\tilde{x})\right)
$$

with $\Re[\cdot]$ denoting the Hardy-Littlewood maximal operator on $\partial \tilde{T}$, and therefore (11) becomes

$$
\begin{aligned}
{\left[u_{2}\right]_{B}^{2} } & <C^{2}\|f\|_{*}^{2} \int_{|\tilde{x}|<1}\left\{\Re[\cdot]\left(\tilde{x}, \varphi_{\delta}(\tilde{x})\right)\right\}^{2}\left\{\int_{\varphi_{\delta}(\tilde{x})}^{\varphi_{\delta}(\tilde{x})+2}\left[\tilde{t}-\varphi_{\delta}(\tilde{x})\right] d \tilde{t}\right\} d \tilde{x} \\
& <C^{2}\|f\|_{*}^{2} \int_{\partial \tilde{T}} \Re[\tilde{K}]^{2} d \tilde{\sigma} \leqslant C^{2}\|f\|_{*}^{2} \int_{\partial \tilde{T}}[\tilde{K}]^{2} d \tilde{\sigma}
\end{aligned}
$$


by the $L^{2}$ boundedness of the maximal operator. Consequently, this estimate and (8) imply that

$$
\|u\|_{* *} \leqslant C\|f\|_{*}
$$

by the $L^{2}$ integrability of $\tilde{K}$.

\section{BIBLIOGRAPHY}

1. B. E. J. Dahlberg, On estimates of harmonic measure, Arch. Rational Mech. Anal. 65 (1977), 275-288.

2. __ On the Poisson integral for Lipschitz and $C^{1}$ domains, Studia Math. (to appear)

3. Weighted norm inequalities for the Lusin area integral and the nontangential maximal function for functions harmonic in a Lipschitz domain, Chalmers Inst. Technology and Univ. of Goteborg, 1977 (preprint).

4. $\_L^{q}$ estimates for Green potentials in Lipschitz domains Tech. Rep., Chalmers Inst. Technology and Univ. of Goteborg, 1977.

5. E. Fabes, R. Johnson and U. Neri, Spaces of harmonic functions representable by Poisson integrals of functions in BMO and $\mathfrak{e}^{p, \lambda}$, Indiana Univ. Math. J. 25 (1976), 159-170.

6. E. Fabes and U. Neri, Harmonic functions with BMO traces Lipschitz curves, Tech. Rep. 78-41, University of Maryland, July, 1978.

7. R. A. Hunt and R. L. Wheeden, On the boundary values of harmonic functions, Trans. Amer. Math. Soc. 132 (1968), 307-322.

8. B. Muckenhoupt and R. L. Wheeden, Weighted bounded mean oscillation and the Hilbert transform, Studia Math. 54 (1975/76), 221-237.

School of Mathematics, University of Minnesota, Minneapolis, Minnesota 55455

Department of Mathematics, University of Maryland, College Park, Maryland 20742 